\title{
Confianza: elemento fundamental en las relaciones entre docentes y directivos
}

\author{
Alexandra Pedraza Ortizi \\ Isabel Muñoz López ${ }^{2}$ \\ José Javier Bermúdez Aponte ${ }^{3}$ \\ Universidad de La Sabana, Colombia
}

Recepción: 15-10-2016 / Aceptación: 03-12-2016

\section{Resumen}

El presente artículo da cuenta de la investigación realizada sobre la confianza entre docentes y directivos en la Institución Educativa Distrital San Francisco (Bogotá, Colombia). Se considera la confianza como factor que repercute en la consolidación de la cultura escolar y en el logro de objetivos institucionales, ya que a su vez, es un elemento mediador de relaciones humanas, al configurarse como la base de las relaciones sociales.

La investigación desarrolló un ejercicio de intervención, en el que instrumentos cuantitativos, permitieron describir los niveles de confianza en las relaciones entre directivos y docentes. Desde allí, se realizó un análisis cualitativo para observar la relación con los procesos académicos y convivenciales de la institución. Los resultados arrojados permiten plantear estrategias pedagógicas y organizacionales para el aumento en los niveles de confianza, el mejoramiento de la calidad educativa y la consolidación de una cultura escolar.

Palabras clave: confianza, relaciones, cultura escolar, procesos institucionales.

\begin{abstract}
This article bears of the carried research about confidence between teachers and principals at the Educational Institution San Francisco District (Bogotá, Colombia.) Confidence is considered as a factor that impacts in the consolidation of the school culture and in the achievement of institutional objective since it turns as a mediating element of human relations, conceiving it like the basis of social relations.

The research developed an exercise of intervention in which quantitative tools allowed the description of levels of confidence in the relations between teachers and principals. From there, a qualitative analysis was carried in order to observe the connection in the academic and harmonic processes of the institution. The obtained results permit the formulation of pedagogical and organizational strategies increasing the levels of confidence, the improvement of educational quality, and the consolidation of a school culture.
\end{abstract}

Key Words: confidence, relations, school culture, institutional processes.

1. Magíster en Educación, Docente, Universidad de La Sabana; email: alexandra.pedraza@unisabana.edu.co

2. Magíster en Dirección y Gestión de Instituciones Educativas, Coordinadora Colegio San Francisco IED; email: isabel-munoz@hotmail.com

3. Doctor en Gobierno y Cultura de las Organizaciones, Universidad de La Sabana;

email: javier.bermudez@unisabana.edu.co 


\section{Introducción}

Las instituciones educativas son organizaciones humanas basadas en relaciones y vínculos entre las personas que las constituyen y orientan sus acciones al logro de objetivos comunes, de acuerdo con su naturaleza institucional. Según Sandoval (2008), las organizaciones educativas contribuyen al perfeccionamiento personal y social. En Colombia, el Ministerio de Educación Nacional establece que las entidades dedicadas al servicio educativo sustentan el proceso de formación permanente, personal, cultural y social, en una concepción integral de la persona humana, de su dignidad, de sus derechos y de sus deberes. En este mismo sentido, Zapata, Gómez y Rojas (2010) señalan que "las condiciones de este proceso hacen que estas instituciones se definan como organizaciones de aprendizaje porque, a partir de la enseñanza-aprendizaje se procura satisfacer parte de las necesidades de generación de conocimiento en el presente y en el futuro" (p. 79).

En las últimas décadas han surgido reflexiones sobre cultura institucional y confianza como un factor importante del capital social y simbólico de las organizaciones, en la medida en que sustenta las relaciones de sus diferentes actores, en su calidad de individuos y en su condición de miembros de los estamentos que las componen (Parra, 2011; Covey y Merril, 2007; Abarca, 2004; Bolívar, 1997; Luhman, 1996). Estas investigaciones señalan la confianza como un componente a considerar en las políticas y estrategias institucionales de toda organización, especialmente en las educativas ya que dentro de sus funciones formativas está: "posibilitar a la persona crecer, mejorar, perfeccionarse, desarrollarse integralmente y trascender" (Sandoval, 2008, p.113), y fundamentan el desarrollo de competencias en valores individuales y sociales, como base para la concreción de proyectos de vida y el desempeño en los entornos sociales.

Desde la perspectiva de las relaciones interpersonales, la confianza se define como "una actitud basada en la buena fe de las acciones de los participantes de una interacción social, para la consecución de fines comunes y la creencia en que los actores comprometidos cumplen sus responsabilidades con autonomía y autorregulación" (Abarca, 2004, p. 58). Dado que las relaciones interpersonales son la base del funcionamiento de las organizaciones, la confianza es el factor que facilita las funciones de los miembros, al tiempo que refleja la existencia y apropiación de los principios y valores de la organización.

De tal modo, dentro de las lógicas que imperan en el desempeño de los miembros de la institución, la confianza tiene una importancia estratégica en el logro de los objetivos y en la racionalización de sus procesos: a mayor confianza menor complejidad de los procesos; y a menor confianza, mayor complejidad de estos (Covey y Merrill, 2007), o en palabras de Conejeros, Rojas y Segura (2010) confiar permite reducir la complejidad social (p. 32). 
En este sentido, las instituciones educativas deben formar en estrategias y valores asociados a la confianza como el respeto, la adecuada comunicación, la integridad, entre otros; así como propender por generar ambientes formativos fundamentados en este valor, pues estos:

Permiten a los seres humanos trabajar en forma individual o en equipo, desarrollar una comunicación abierta, compartir información y exponer puntos de vista sin temor a que se les menosprecie o subestime, lo que constituye, en sí mismo, un ambiente propicio para el aprendizaje a todo nivel (Zapata et al., 2010, p. 82).

A partir de lo anterior, la investigación realizó un análisis de la percepción de la confianza en las relaciones entre docentes y directivos del colegio San Francisco IED, de la localidad de Ciudad Bolívar, ubicado en Bogotá, Colombia. En esta se reconoce la confianza como un factor que media en las interacciones de estos actores educativos y en la apropiación de los principios y valores institucionales.

Es importante tener en cuenta que la comunidad educativa se ve afectada por problemas recurrentes como deficiencias en comunicación, apatía de los docentes por instrucciones e indicaciones, contradicciones entre discurso y práctica, falta de sentido de pertenencia y falta de liderazgo de algunas instancias directivas; manifestaciones que sugieren bajo nivel o ausencia de confianza.
Por tal razón, es importante resaltar que las relaciones personales e interpersonales deben estar mediadas por la confianza, puesto que ella permite establecer un concepto de comunidad, da vitalidad y en algunos casos, revitaliza, el carácter de solidaridad de la colectividad; y es que en este aspecto, la comunidad permite que el individuo crezca, adquiera identidad, sentido de pertenencia y potencie sus posibilidades y virtudes.

En este sentido, las confianza como valor y virtud de los individuos, para gestar comunidad, se establece como necesaria en el ámbito educativo; toda vez que la escuela es la manifestación más clara de la sociedad. Por ello, la confianza debe constituirse en elemento vital para consolidar la escuela como escenario de aprendizaje y construcción de sociedades más justas y equitativas,

el establecimiento y fomento de la confianza es fundamental para reestructurar la educación. El reto de la confianza consiste en reconstruir unas relaciones de trabajo en colaboración entre colegas próximos que refuercen la significación personal, sin fortalecer el paternalismo y el localismo" (Hargreaves, 1995, p. 279).

En el ámbito educativo, los directivos, como líderes, son los hacedores de los criterios y estructura de la gestión escolar. Ellos deben identificar oportunidades, amenazas, cambios y tendencias en su entorno, para adaptar la organización a los constantes retos que las 
directrices locales, nacionales e internacionales les exigen (Veciano, 1999). Asimismo, deben tomar decisiones mostrando entereza y convencimiento sobre la razón de estas; igualmente, $\mathrm{y}$ han de promover la participación de sus colaboradores.

En ese sentido, para Cardona (2006) es muy importante que los directivos desarrollen la dirección por virtudes, sean profesionales coherentes en su pensar y actuar, se conozcan a sí mismos, y que a la hora de dirigir, contemplen el ayudar y desarrollar los potenciales de los demás. El autor afirma que "un buen directivo es capaz de generar expectativas elevadas constantemente. Tiene confianza en su capacidad personal para desarrollar el talento de su gente" (Cardona, 2006, p. 94). Por otro lado, están los docentes, quienes junto con los directivos son piezas claves en las dinámicas educativas. En ellos se gesta el sistema organizacional de la institución, son quienes lideran el aula y de ellos surge la cultura escolar con ritos y acciones.

Ellos deben recibir de sus directivos, la confianza e impulso para generar relaciones positivas que sean ejemplo ante sus estudiantes y la comunidad educativa en general.

Como marco para fortalecer la relación de confianza entre directivos y docentes, se tienen en cuenta las consideraciones de Cardona (2002), quien propone algunos elementos que pueden cohesionar la confianza entre las personas:

1. La ética: entendida como la forma de interactuar entre los otros, con valores y virtudes como la honestidad, la imparcialidad, el no mentir, el cumplir con la palabra que se da, el ser coherente y responsable. / 2. La madurez: alcanzar un grado de autoconciencia que permite actuar de manera coherente con los requerimientos del entorno, es no obstaculizar el buen criterio de la empresa. / 3. El interés y la disponibilidad: es estar accesible a los demás, es estar presto a donarse a ayudar a otras personas. / 4. La credibilidad: cuando las demás personas perciben que se es íntegro, que se cumple con la palabra dada. / 5. Competencia profesional: es definir estrategias, establecer objetivos, delegar, evaluar el rendimiento, aplicar las políticas, resolver conflictos, tomar decisiones, ser competente. / 6. Admitir críticas constructivas. Ejercer la disciplina correctamente: el directivo eficaz que consolida la confianza y crea compromisos debe manejar una comunicación adecuada con el propósito de mejorar los desempeños y el logro de los objetivos en sus colaboradores (p. 60).

De acuerdo con lo anterior, es indispensable que se piense en las habilidades necesarias para fomentar la confianza entre los directivos y docentes; además pensar en si estas relaciones se centran en la confianza potenciando la calidad humana, como lo afirma Guillen (2006): "cuando se habla de calidad humana es respetar al otro, es potenciar cualidades como la flexibilidad, sinceridad, transparencia, orden, optimismo, lealtad, generosidad esto incide directamente en el comportamiento del propio individuo y de los que lo rodean generando confianza" (2006, p. 11).

El directivo puede ejercer su liderazgo, y cumplir con los objetivos de la institución, si 
comunica los criterios con los que toma las decisiones, delega las responsabilidades en quien debe y puede asumirlas; esta dimensión de la confianza es de orden ético, la cual es condición básica para que las relaciones de autoridad y subordinación sean fluidas y estables todo el tiempo (Guillen, 2006).
Cuando se confía en quien lidera y en el equipo de trabajo, los compromisos adquiridos son asumidos con mayor responsabilidad, la lealtad se ve reforzada. Esto se puede representar en un cuadrante que claramente muestra todo lo que se desencadena cuando se potencializa la calidad humana (ver figura 1).

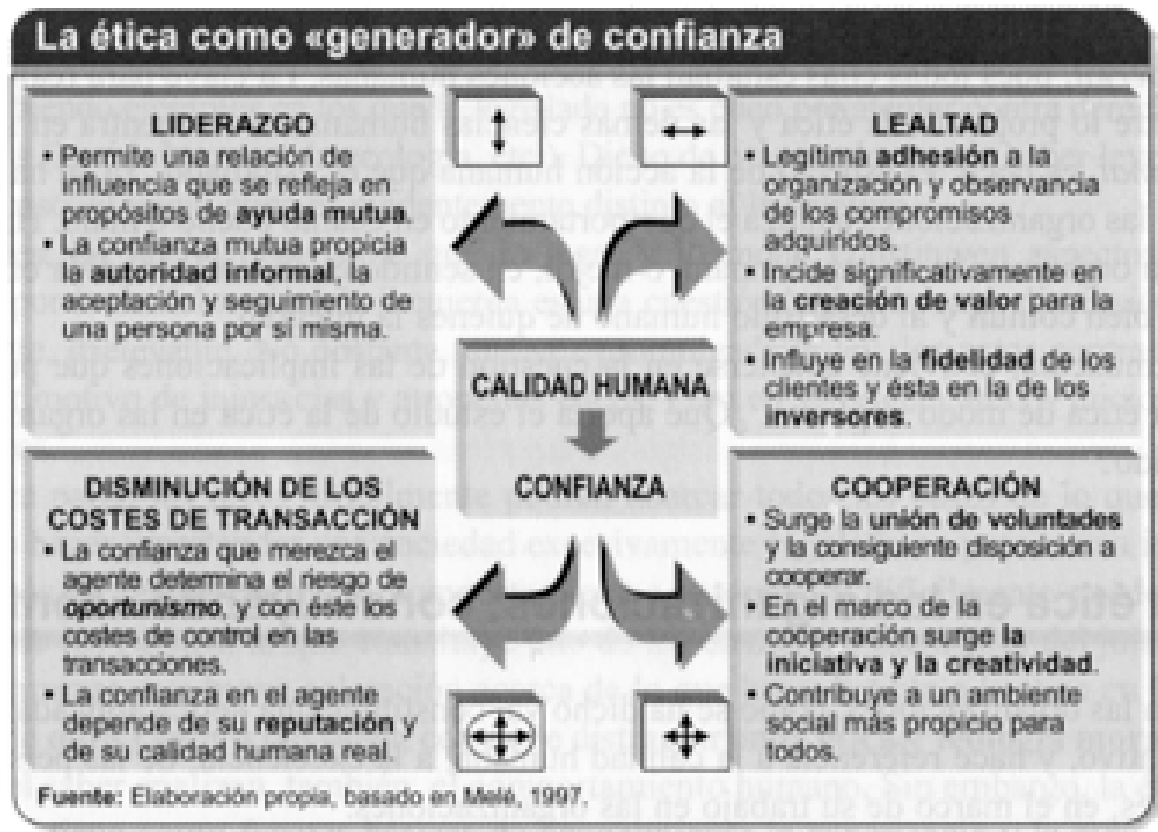

Figura 1. Cuadrante de la ética.

Fuente: Guillén, 2006.

Para fomentar la confianza en cualquier tipo de relación, bien sea de tipo personal o profesional, Covey y Merrill (2007) proponen fomentar trece conductas, las cuales tienen que ver con el carácter y las competencias y su unión para crear un equilibrio. Éstas pretenden cambiar paradigmas en la forma de ver y de pensar, lo que contribuye al logro de los objetivos, no solo personales, sino de la institución donde se labora. A continuación se presentan estas trece conductas que son la base para hacer ejercicio de intervención en la presente investigación:

a. Hablar claro: ser honesto, decir la verdad; se basa en los principios de integridad, honestidad y franqueza. Emplear un lenguaje sencillo.

b. Demostrar respeto: demuestra la educación de una persona, es demostrar amabilidad, amor y civismo. 
c. Crear transparencia: ser abierto, veraz y sincero, decir la verdad, con ello la confianza se gana más rápidamente.

d. Corregir errores: esta conducta implica más que disculparse, reconciliarse. Es pasar a la acción, se basa en los principios de humildad, integridad y compensación.

e. Mostrar lealtad: se basa en los principios de integridad, gratitud, reconocimiento. Cuando se le reconoce el mérito a otro, se le anima a participar, a ser innovador, a colaborar.

f. Presentar resultados: los resultados dan credibilidad y confianza al instante, dan influencia, demuestran claramente que se puede aportar, contribuir, rendir en la tarea asignada. Esta conducta surge de los principios de responsabilidad, compromiso, rendimiento.

g. Mejorar: se basa en los principios de la mejora continua, aprendizaje y cambio; para ello, hay dos estrategias fundamentales: buscar opiniones y aprender de los errores.

h. Afrontar la realidad: se requiere el principio de valor, responsabilidad, conciencia y respeto.

i. Clarificar las expectativas: consiste en crear una visión compartida y llegar a acuerdos por adelantado sobre lo qué se va a hacer, se deben tener compromisos y acuerdos claros. j. Practicar la responsabilidad: basa en maximizar la responsabilidad y la obligación.

k. Escuchar primero: significa escuchar de verdad (entender pensamientos, sentimientos, expectativas, experiencia $y$ puntos de vista del otro); es de vital importancia tratar de entender primero para evitar suposiciones.

1. Mantener los compromisos: esta conducta se basa en los principios de integralidad, rendimiento, valor y humildad.

m. Ampliar la confianza: cuando se aplican las anteriores doce conductas, se está preparado para ampliar la confianza. Esta conducta se basa en los principios de reciprocidad y la creencia fundamental de que las personas son dignas de confianza.

Cuando se amplía la confianza se debe trabajar en los cuatro principios de credibilidad: integridad, intensiones, capacidades y resultados; se aumenta el valor a lo que se haga (integridad), se fomentan las predisposiciones hacia la confianza (intenciones), se mejora la capacidad de clarificar expectativas, se logra que los demás cumplan sus compromisos (capacidades), de esta manera se mejoran los resultados y se transforma la cultura organizacional (Covey y Merrill, 2007).

Para mejorar la intención organizacional en la institución, el directivo debe asegurarse que la misión y los valores reflejen principios que fomenten la confianza. Además, se debe garantizar que los sistemas de información y 
la toma de decisiones estén alineados con los esfuerzos por satisfacer las necesidades institucionales y de la comunidad. Para mejorar los resultados, también se puede generar un plan de acción compartido con los entes del gobierno escolar, en el que se incluyan objetivos graduales, metas a corto, mediano y largo plazo; esto hará que la institución se consolide bajo una nueva mirada: la confianza.

\section{Metodología}

La investigación realizada fue de naturaleza descriptiva, que de acuerdo con Hernández, Fernández y Baptista (2003), busca especificar las propiedades importantes y relevantes de un objeto de estudio; también, busca medir o evaluar los aspectos, dimensiones o componentes relevantes del fenómeno a investigar. La investigación propone la resolución de problemas, que "está basado en procesos cognitivos que tienen como resultado encontrar una salida a una dificultad, una vía alrededor de un obstáculo, alcanzando un objetivo que no está inmediatamente alcanzable" (Mayer, 1983, p. 21).

Para desarrollarla, se partió de la realización de un diagnóstico sobre las percepciones de confianza entre docentes y directivos del colegio San Francisco IED de la localidad de Ciudad Bolívar, y su incidencia en los procesos que se dan al interior de la misma, enmarcadas en las categorías propuestas por Covey y Merrill (2007). Con dichos resultados se propuso una intervención para potenciar la confianza como elemento dinamizador de las relaciones entre los docentes y los directivos.
Esta investigación tuvo como base un enfoque cualitativo, que buscó examinar el mundo social y en ese proceso desarrolla una teoría coherente con lo que observa y ocurre. Desde esta perspectiva, el análisis propone comprender una realidad con argumentos teóricos que intentan explicarla partiendo de la premisa de que los niveles de confianza influyen en la forma como las personas establecen las relaciones; resultando así, un factor determinante en las relaciones interpersonales entre docentes y directivos, lo cual contribuye a dinamizar los procesos que se dan en una institución educativa para el logro de sus fines.

Los participantes fueron 38 docentes que, por condiciones de acceso y decisión voluntaria, colaboraron con el estudio (de un total de 105), y a la totalidad de los directivos de la institución de sus tres sedes y jornadas (6 directivos y 7 orientadores).

Las categorías de análisis que se reconocieron para el estudio fueron las planteadas por Covey y Merrill (2007), que proponen siete niveles del estado de la confianza en las organizaciones que se desprenden de las condiciones del entorno institucional y de las lógicas y principios de las relaciones personales, las cuales son:

a. Confianza inexistente. Existencia de un entorno disfuncional y cultura tóxica, dirección muy centralizada y fiscalizadora, jerarquía redundante; en cuanto a las relaciones personales se destacan los enfrentamientos acalorados, las poses defensivas, los calificativos de enemigos, y la comunicación es agresiva. 
b. Confianza muy escasa. Entorno de trabajo malsano, descontento por los trabajadores, ambiente marcado por bandos, excesivo tiempo malgastado en la defensa de posiciones y decisiones; con respecto a las relaciones personales se presentan conductas hostiles, gritos, reproches, acusaciones, insultos, preocupaciones y sospechas contantes, los errores se utilizan como armas.

c. Confianza escasa. Habituales conductas para cubrirse las espaldas, hay intenciones ocultas, bandos políticos con aliados y enemigos; en cuanto a las relaciones personales, predominan las interacciones faltas de alegría, se pone en tela de juicio la fiabilidad y el compromiso de los demás.

d. Problemas de confianza. Caracteriza a la organización con jerarquías innecesarias, aprobaciones lentas, desajuste en sistemas y estructuras, algunos colaboradores insatisfechos; con respecto a las relaciones personales, se presentan malentendidos habituales, preocupaciones por intenciones y motivaciones, interacciones tensas, comunicación influida por el miedo y la incertidumbre.

e. La confianza no es un problema. Lugar saludable para trabajar, con un sistema y estructuras organizadas, se presentan pocas intrigas de oficina; a nivel de relaciones personales, se presentan comunicaciones cordiales y saludables, se prioriza trabajar juntos con fluidez y eficacia, prevalece la tolerancia y la aceptación mutua. f. La confianza es un activo destacado. La prioridad es el trabajo, hay colaboración y ejecuciones efectivas, relaciones de asociación positivas, creatividad e innovación sólidas; con respecto a las relaciones personales se presenta la cooperación, la prioridad es buscar y aprovechar los puntos fuertes de cada uno y la comunicación es edificante y positiva.

g. Confianza de calidad superior. Elevados niveles de colaboración y asociación, comunicación sin esfuerzo, relaciones positivas, transparentes, sistemas y estructuras totalmente ajustados, innovación compromiso, confianza y lealtad sólida, trabajo inspirador.

Cada uno de estos estados se mide de acuerdo con indicadores basados en la percepción de los actores de la organización; en este caso los docentes y directivos que dieron su punto de vista sobre la institución.

De acuerdo con los autores mencionados, el estado de la confianza en la organización tiene impacto en la economía de las organizaciones, en la medida en la que "al reducirse la confianza, también se reduce la rapidez y se incrementan los costes. Cuando se incrementa la confianza, también se incrementa la rapidez y se reducen los costes"(Covey y Merrill, 2007, p. 39), de tal forma que los primeros cuatro estados: confianza inexistente, confianza muy escasa, confianza escasa y problemas de confianza les generan impuestos a las organizaciones que gravan las actividades y servicios de esta, también, "todas las activi- 
dades: en todas las relaciones, en todas las interacciones, en todas las comunicaciones, en todas las decisiones, en todas las dimensiones de la vida" (Covey y Merrill, 2007, p. 46).

En tanto los otros tres estados: la confianza no es un problema, la confianza es un activo destacado y la confianza de calidad superior generan dividendos, puesto que "en una empresa, un buen nivel de confianza mejora sustancialmente la comunicación, la colaboración, la ejecución, la innovación, la estrategia, el compromiso, la asociación y las relaciones en todas las partes interesadas" (Covey y Merrill, 2007).

Luego, se propone una segunda categorización para determinar los niveles de credibilidad en la organización que es el principio básico de la confianza en la organización, según Covey y Merrill (2007). Estos autores proponen cuatro focos para observar la credibilidad que son: la integridad que es la coherencia entre el actuar y el decir, es actuar bajo un esquema de valores y creencias no importando las circunstancias del momento; las intenciones que tienen que ver con las motivaciones, las prioridades y conductas resultantes; las capacidades son las aptitudes, talentos, actitudes, destrezas, conocimientos, y estilos que se utilizan para alcanzar los resultados, a nivel de organizaciones y los resultados que se refieren al rendimiento; es conseguir el logro de los objetivos propuestos y alcanzar las metas asignadas.

\section{Resultados}

$\mathrm{Al}$ aplicar el instrumento de investigación se pudieron determinar los impuestos (los costos que genera un bajo nivel de confianza en la institución) y los dividendos (los beneficios que generan un alto nivel de confianza).

Analizando las respuestas de la encuesta, en relación con las categorías propuestas que permiten identificar los siete tipos de estados de la confianza: confianza inexistente (A), confianza muy escasa (B), confianza escasa (C), problemas de confianza (D), la confianza no es un problema (E), la confianza es un activo destacado (F), confianza de calidad superior $(G)$, se puede determinar que los

Tabla 1. Nivel de confianza en la organización y en las relaciones personales. En la organización/ En las relaciones personales

\begin{tabular}{|c|c|c|}
\hline Niveles de confianza & Número de personas & Porcentaje \\
\hline Inexistente & 1 & $2.3 \%$ \\
\hline Muy escasa & 4 & $9.3 \%$ \\
\hline Escasa & 7 & $16.2 \%$ \\
\hline Problemas de confianza & 25 & $58.2 \%$ \\
\hline No es un problema & 4 & $9.3 \%$ \\
\hline Es un activo destacado & 2 & $4.6 \%$ \\
\hline Calidad superior & 0 & $0 \%$ \\
\hline
\end{tabular}


impuestos y dividendos se distribuyen en los porcentajes que se observan en la tabla 1.

Por lo anterior, se puede afirmar que la percepción del $85.8 \%$ de los encuestados con respecto al estado de la confianza, corresponde a nivel organizacional de disfuncionalidad, en donde hay jerarquías innecesarias, aprobaciones lentas en los procesos, no hay una alineación clara entre la misión y la visión institucional; se constituye oportunidad de mejora en la relación entre los valores institucionales y las prácticas cotidianas, planes de trabajo claros y en el seguimiento a procesos. En cuanto a las relaciones personales, se presenta la necesidad de fortalecerlas, ya que hay malentendidos habituales y deficiencia en la comunicación. Lo anterior indica un estado de confianza muy escasa, que implica impuestos para el funcionamiento de la organización y bajos dividendos.

En la segunda parte del instrumento se determinaron los niveles de credibilidad que se viven en la institución y con ello el grado de confianza que se vive en la organización. La credibilidad es un concepto utilizado por las personas para determinar si creen o no en una información brindada, de la que no son testigos. Para Covey y Merrill (2007) esta se sustenta en cuatro pilares bases o cuatro variables que son integridad, intenciones, capacidades y resultados. La credibilidad va de la mano con la verdad, se es más confiable en cuanto se es más creíble.

Esto quiere decir que cuanto más fuerte sea la percepción de cada uno de los pilares de la credibilidad al interior de la institución, mayor será el nivel de confianza entre sus integrantes.

La integridad, según Covey y Merrill (2007) es la coherencia entre el actuar y el decir, es actuar bajo un esquema de valores y creencias no importando las circunstancias del momento. De acuerdo con ello, se puede decir que el $88 \%$ de los encuestados centra sus respuestas en situaciones negativas, afirmando que se vive un bajo nivel del valor de la honestidad en las actuaciones, que falta coherencia entre en el decir y en el actuar, no hay claridad en los valores institucionales que se viven en las prácticas, se dificulta la disposición de aceptar nuevas ideas que inviten a replantear prácticas e incluso valores, y no se vive una cultura del compromiso.

La figura 2 evidencia la percepción que los encuestados tienen de la integridad de los demás miembros de la institución, en la cual el $88 \%$ considera que la integridad es débil, mientras que un porcentaje increíblemente bajo del $0.5 \%$ considera que la integridad es fuerte.

Las intenciones tienen que ver con las motivaciones, las prioridades y conductas resultantes. La confianza se da cuando las intenciones son claras y se basan en el beneficio mutuo, en la cooperación, en el compartir abiertamente las ideas y la información; la integridad y las intenciones tienen que ver con el carácter, ya sea de una persona o de una organización. Este pilar está conformado, también, por cinco variables en donde se describen situaciones relacionadas con la preocupación por el bienestar del otro, por la motivaciones y las 


\section{Integridad}

$\square 1$ Fuerte $\square 2 \square 3 \square 4 \square 5$ Débil

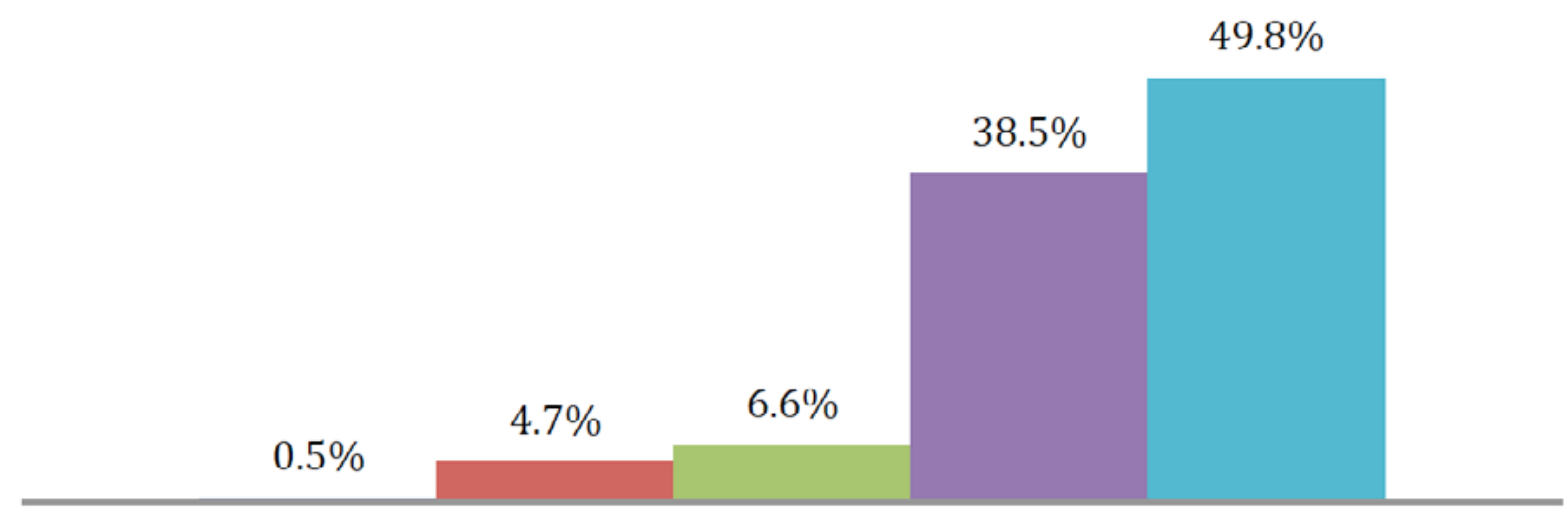

Porcentaje de percepción de integridad

Figura 2. Gráfico de calificación de la integridad.

actuaciones correctas, por la relación ganarganar frente a la toma de decisiones, teniendo la opción de escoger de 1 a 5 , en donde el 1 es la conducta ideal.

En este pilar, el 55\% de los encuestados señalan que hay una cultura de poca preocupación por el otro, es necesario mejorar la posición de indiferencia frente al bienestar del otro, hay poca motivación para perfeccionarse y hacer las cosas mejor; se está pensando de manera individual, que se hacen la cosas, casi siempre, por los intereses de las áreas (ver figura 3).

Las capacidades son las aptitudes, talentos, actitudes, destrezas, conocimientos y estilos que se utilizan para alcanzar los resultados a nivel de organizaciones. Covey y Merrill (2007) plantean la necesidad de evaluar si tienen los medios para prestar un servicio de calidad y competir en el mercado; la capacidad de potenciar el talento humano de la organización, de mejorar e innovar continuamente. Este pilar consta de cuatro variables, que hablan sobre la correspondencia entre los talentos y las oportunidades para demostrarlas en el trabajo que se realiza, actualización de conocimientos, la eficacia de utilizar las fortalezas que se tienen y la preocupación por cultivar, difundir y ampliar la confianza.

Los encuestados, en relación a este aspecto, señalan que no hay coherencia entre los talentos y las oportunidades de trabajo; pocas personas se preocupan por la capacitación y la actualización, no es importante identificar las oportunidades de mejora para trabajar en ellas, no se cultiva y desarrolla la confianza y no se sabe cómo hacerlo (ver figura 4). 


\section{Intenciones}

$\square 1$ Fuerte $\square 2 \square 3 \square 4 \square 5$ Débil

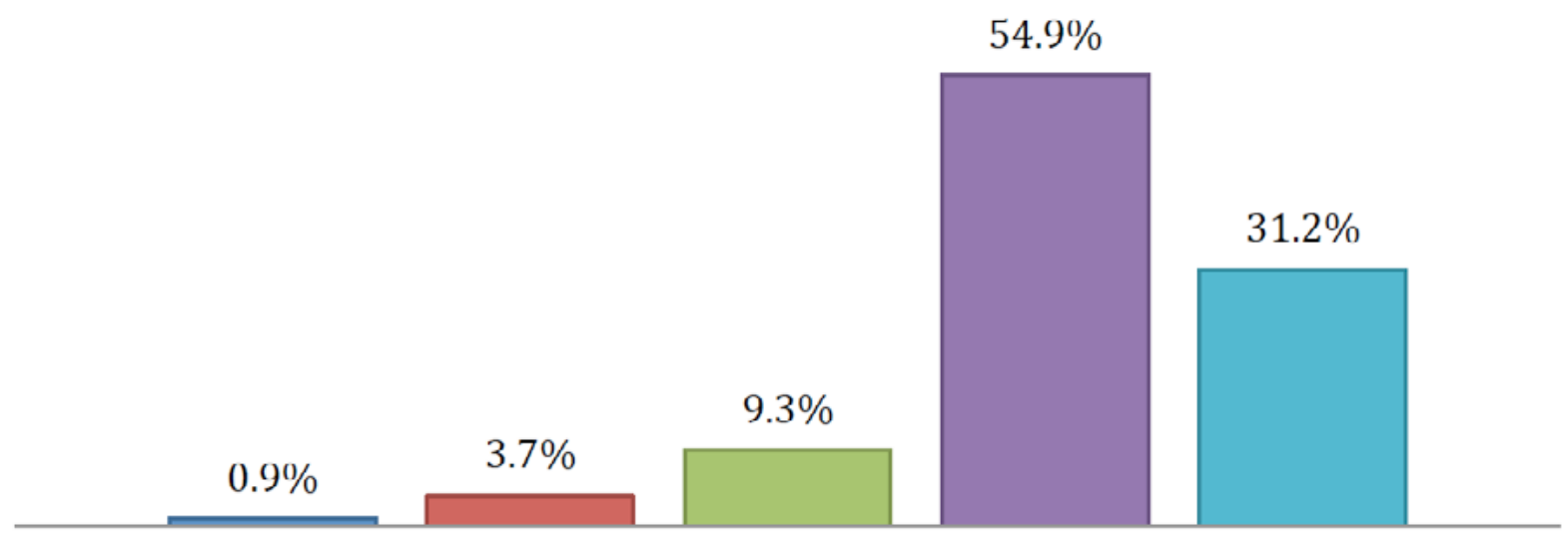

Porcentaje de percepción de las intenciones

Figura 3. Gráfico de calificación de las intenciones.

\section{Capacidades}

a Fuerte $\quad 2 \quad \square 3 \quad 4 \quad \square 5$ Débil

$46.5 \%$

$11.6 \%$

$4.7 \%$

$0.6 \%$

Figura 4. Gráfico de calificación de las capacidades. 
Covey y Merrill (2007) se refieren a los resultados cuando se habla de trayectoria, de rendimiento; es conseguir el logro de los objetivos propuestos y alcanzar las metas asignadas. Este pilar consta de cinco variables, que plantean la importancia de la trayectoria de los resultados, los esfuerzos se concentran en los resultados más no en las actividades, la trayectoria de la institución en cuanto a resultados que inspiran confianza, se termina lo que se empieza, hay una sistematización en el logro de los resultados.

A este respecto, los encuestados afirman que la organización no logra los resultados propuestos en su totalidad; las personas centran sus esfuerzos en las actividades aisladas, falta un seguimiento sistematizado que evidencie la consecución de los resultados, se presentan dificultades que afectan el cumplimiento en el cronograma propuesto; reina la postergación en la mayoría de los procesos, y que para la institución no es relevante cumplir con lo que se ha propuesto año a año (ver figura 5).
El análisis de las calificaciones dadas permite afirmar que el $87 \%$ de los encuestados tienen la percepción de que en el colegio se vive una cultura de poca credibilidad, lo cual se evidencia al comparar los cuatro pilares que la conforman: integridad, intenciones, capacidades y resultados (ver figura 6).

Para concluir, se puede inferir que, según los docentes y los directivos encuestados se evidencia una percepción de falta de comunicación asertiva; se vivencia una cultura en la que prima el bienestar propio, falta una alineación entre las prácticas pedagógicas, los valores institucionales y los valores personales; no hay una necesidad sentida de mejorar los resultados, se presenta ausencia de políticas claras dentro de la institución, que permitan la alineación del horizonte institucional con las prácticas del día a día, escasos seguimientos a los procesos, y se vive una cultura tóxica que no permite avanzar (ver figura 7).

\section{Resultados}

$\square 1$ Fuerte $\square 2 \square 3 \square 4 \quad \square 5$ Débil

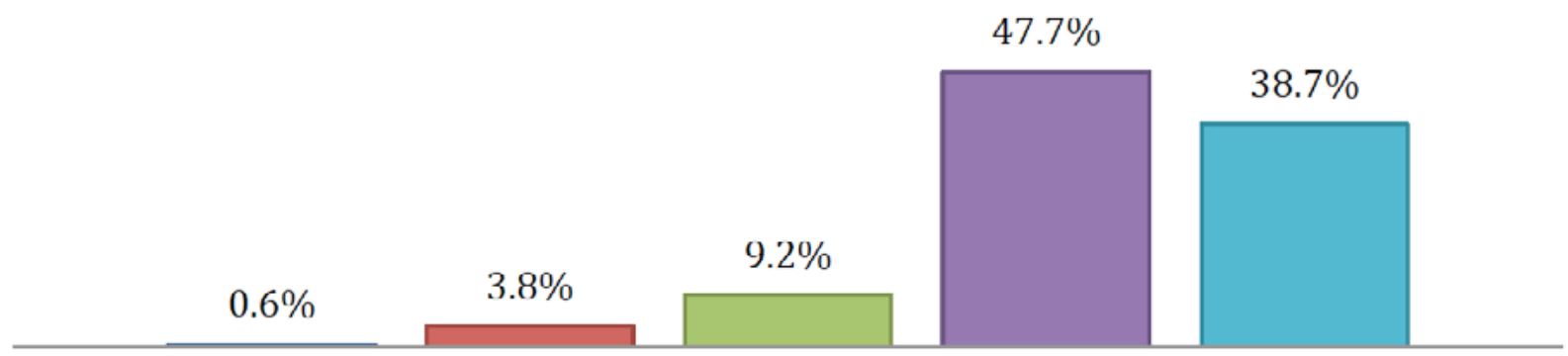

Porcentaje de percepción de resultados

Figura 5. Porcentajes de calificación de los resultados. 


\section{Credibilidad}

$\square 1$ Fuerte $\square 2 \square 3 \quad \square 4 \square 5$ Débil

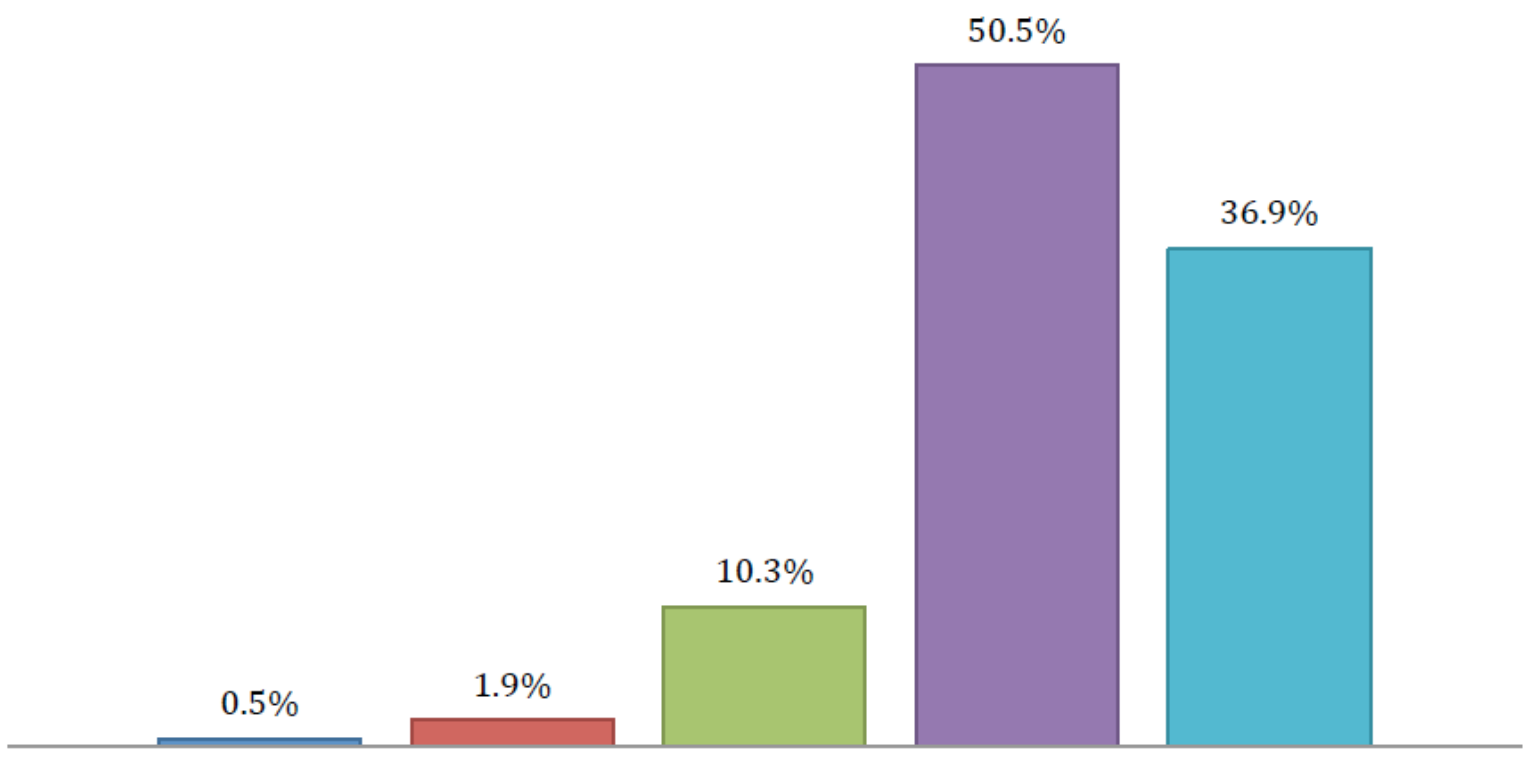

Porcentaje de percepción de credibilidad

Figura 6. Gráfica de calificación global de la credibilidad.

\section{Confianza}

1 Fuerte $\square 2 \square 3 \square 4 \square 5$ Débil

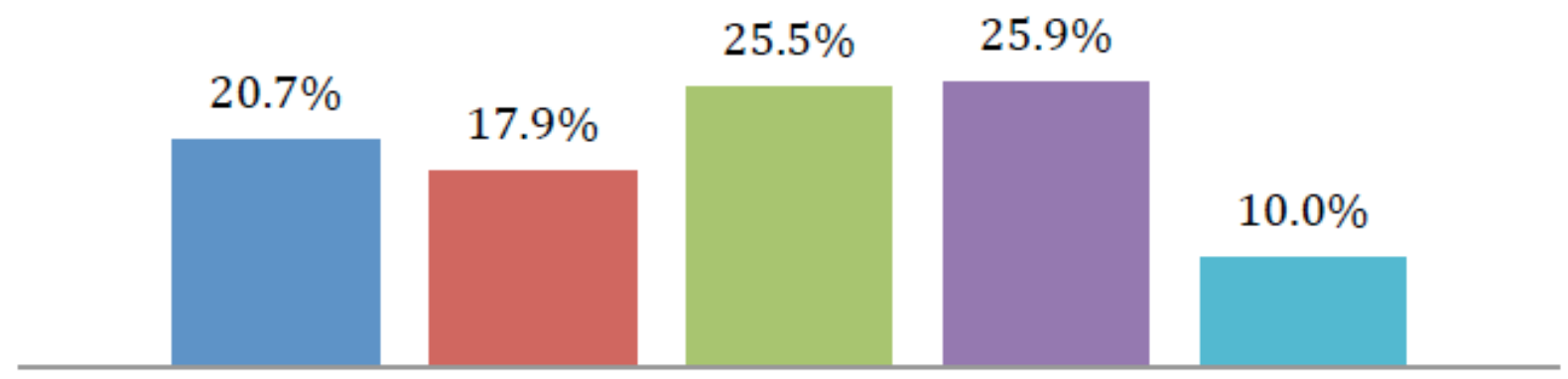

Porcentaje de percepción de confianza

Figura 7. Percepción global de la confianza en las relaciones. 


\section{Discusión}

Según Covey y Merrill (2007), cuando en una institución hay bajo nivel de confianza, generalmente, se presentan comportamientos como: a) hechos distorsionados o manipulados, b) se presentan dificultades en el manejo de la información, c) las personas muestran la verdad a su conveniencia, d) recibir elogios es lo principal, e) se presenta resistencia ante las nuevas ideas, ante los proyectos de innovación, f) se ocultan los errores, g) la mayoría de las personas entran en el juego de minimizar a otros, h) hay comentarios de pasillo, i) hay reuniones poco productivas, $j$ ) se presentan muchas expectativas sin cumplir, hay muchas excusas, k) algunas personas fingen que no están sucediendo cosas negativas, $y$ 1) las personas a menudo sienten tensión improductiva; incluso miedo.

De allí, la importancia de realizar un ejercicio de intervención con el que se demuestre a los docentes y directivos la necesidad de trabajar estos cuatro focos, para que la institución tenga credibilidad en sus procesos y la confianza necesaria para el éxito en sus prácticas, conductas y resultados; entendiendo las conductas como una manifestación de lo que se dice y lo que realmente se vive en una cultura como afirman Covey y Merrill:

Las buenas palabras tienen su lugar. Indican una conducta. Declaran intenciones. Pueden crear una esperanza enorme. Y cuando a esas palabras las respalda una conducta que la valide aumenta la confianza, a veces de manera espectacular. Pero cuando las conductas no respaldan o no casa con el lenguaje verbal, las palabras se convierten en una marcha atrás (2007, p. 193).

A su vez, se busca contribuir al cambio de cultura que se vive en la institución en pro de mejorar los procesos organizacionales, el empoderamiento de las directivas, la mejora los resultados académicos y convivenciales y las relaciones que allí se dan a través de la confianza.

Una vez aplicado el instrumento de recolección de datos y determinada la percepción de la confianza en la institución, se organizó un ejercicio de intervención teniendo en cuenta lo planteado por Covey y Merrill (2007). La estrategia de intervención busca el reconocimiento y el afianzamiento del factor confianza en la institución, con lo cual puede sustentar un plan de mejoramiento direccionado a la cultura institucional (Ministerio de Educación Nacional, 2008, p. 28). Para ello se tomó como referente la Guía 34: "Guía para el mejoramiento institucional: de la autoevaluación al plan de mejoramiento" del Ministerio de Educación Nacional (2008).

La propuesta muestra los focos de credibilidad, su definición y ejercicios se pueden proyectar y potenciar la confianza personal y la confianza en la institución, incluyendo las trece actividades que proponen Covey y Merrill (2007) para aumentar la confianza. Se trabajó en el trascurso de seis meses con el propósito de que todos los estamentos de la comunidad la conocieran, y así contribuir a una red de cultura de confianza en la institución. 
La organización educativa se caracteriza como organización del aprendizaje, que desarrolla procesos de formación personal, cultural y social, apoyados en una concepción integral de la persona, su dignidad, derechos y deberes; concepción orientada por el Estado colombiano. Se demostró que la confianza es un componente a tener en cuenta en las políticas y estrategias, y en los planes de mejoramiento para corresponder a las exigencias de su misión institucional.

Asimismo, se sustentó que la confianza es una virtud necesaria para restablecer relaciones y para consolidar todo proceso, dado que es base de ayuda y reciprocidad, pues contribuye al concepto de comunidad, vitaliza la solidaridad y permite crecer como personas, adquirir identidad y sentido de pertenencia.

Los resultados de la investigación permiten realizar reflexiones sobre la confianza como factor dinamizador en las relaciones interpersonales, pues es el vínculo educativo que se construye desde las relaciones con otros: ampliar la confianza a los demás aviva el espíritu interior, ilumina la predisposición innata a confiar y ser digno de confianza; aporta felicidad a las relaciones, resultados en el trabajo y seguridad en la vida y produce un extraordinario dividendo en todas las dimensiones de nuestra existencia. Además, cuando se confía en el otro, se ejecuta un acto de coexistencia aprendiendo a ser en y con los demás.

La libertad, en su sentido pleno, desemboca en la condición radical de la persona que es la coexistencia; afirmando que para ésta existir es propiamente coexistir; que el ser de la persona es ser-con. Cabe decir, aún con mayor radicalidad y plenitud, que el ser de la persona es co-ser-con y, por tanto, carencia de culminación, es decir, que la coexistencia está siempre por alcanzar: la persona. (RodríguezSedano, 2009, p. 32).

Al potenciar la confianza personal se tienen más elementos de valor para mejorar dicha relación de coexistencia y así crecer juntos, este crecimiento trasciende en los objetivos institucionales, pues mejora la comunicación, la empatía, la solidaridad, la aceptación.

La estrategia implementada ofreció un escenario para orientar aspectos hacia el reconocimiento y la interpretación del factor confianza en la institución, con lo cual se pueden sustentar aspectos del plan de mejoramiento direccionados al fomento de una cultura de confianza para apoyar la gestión directiva en el reconocimiento, y legitimidad de las acciones institucionales; la cualificación de los mecanismos de comunicación, la potenciación del trabajo en equipo y la identificación y divulgación de buenas prácticas organizacionales. Todo esto redunda en un clima institucional sano para la convivencia y participación de los integrantes de la institución en los objetivos formativos que la sustentan.

En adición, se recomienda organizar un plan de bienestar, que involucre a docentes y directivos de diversos colegios, con el propósito de seguir trabajando la confianza como elemento dinamizador de relaciones organizacionales. 


\section{Referencias}

Abarca, N. (2004). Inteligencia emocional en el liderazgo. Santiago: Aguilar.

Bolívar, A. (1997). Cambio educativo y cultura escolar. Resistencia y reconstrucción. Innovación educativa, 2, p. 13-22.

Cardona, J. (2002). Del miedo a la confianza. Desarróllese como directivo. Barcelona: Díaz de Santos.

Cardona, J. (2006). Liderazgo y gestión por ocho hábitos. Del miedo a la confianza. Barcelona. Díaz de Santos.

Colombia, Ministerio de Educación Nacional. (2008). Guía 34: Guía para el mejoramiento institucional. De la autoevaluación al plan de mejoramiento. Bogotá: Autor.

Conejeros, M., Rojas, J. y Segura, T. (2010). Confianza. Un valor necesario y ausente en la educación chilena. Perfiles Educativos, 129 (32), 30-46. Recuperado de http://www.scielo.org.mx/ pdf/peredu/v32n129/v32n129a3.pdf

Covey, S. y Merrill, R. (2007). El Factor Confianza. El valor que lo cambia todo. Barcelona: Planeta.

Guillén, M. (2006). Ética en las organizaciones. Construyendo confianza. Madrid: Pearson Educación S.A.

Hargreaves, A. (1995). Profesorado, cultura y postmodernidad. Cambian los tiempos, cambia el profesorado. Madrid: Morata.

Hernández R., Fernández C. y Baptista P. (2003). Metodología de la investigación (3a ed.). México: Mc Graw Hill.

Luhman, N. (1996). Confianza. Nuevo México: Anthropos.

Parra, M. (2011). Ética en las organizaciones. Madrid: Pearson Educación.

Rodríguez-Sedano. (2009). La libre donación personal: libertad íntima y libre manifestación humana desde la filosofía de la educación de Leopoldo Polo. Cuadernos de Anuario Filosófico, 214, 89-98.

Sandoval, E. (2008). Institución educativa y empresa. Navarra: Eunsa.

Veciano, J.C. (1999). Función Directiva. Barcelona: UAB.

Zapata-Jaramillo, C.-M. y Rojas-López, M.-D. (2010). Una revisión crítica al modelado de la confianza a nivel organizacional. Estudios Gerenciales, Recuperado de http://www.scielo.org. co/pdf/eg/v26n116/v26n116a10.pdf 